tissues of 37 HCC patients (20 MVI+ and 17 MVI-). We also investigated DNA methylation profiles of plasma samples from these 37 HCC patients and 30 normal individuals as controls to minimize the interference of random background DNA methylation signals. Tissue and plasma samples were prepared into DNA methylation library and sequenced on Illumina Hiseq X10 platform. Using methylation haplotype load (MHL) and unmethylation haplotype load (UMHL) as metrics, we quantified DNA methylation profiles on methylation haplotype blocks (MHBs) by computing the degree of linkage between methylated or unmethylated CpGs in HCC and adjacent normal samples to identify discriminatory markers. Grouping samples from our cohort into training and validation sets respectively, we employed two supervised machine learning algorithms, random forest (RF) and support vector machine (SVM) to train and cross-validate binary predictive models.

Results Supervised analyses identified 65 MHBs as classifiers for HCC tissues and adjacent normal liver tissues. Both RFand SVM-built models were highly accurate in classifying HCC and normal liver tissues with AUC no less than 0.98 (AUC: 98\%, CI: 97.3\% 98.8\% for RF model; AUC: 99.9\%, CI: $99.9 \sim 99.9 \%$ for SVM model). We applied the RFtrained classification model to differentiate HCC plasma DNA from those of healthy controls, with AUC of 96\% (CI: $95.1 \%$ - 96.9\%). We further identified 6 MHL-quantified MHBs and 5 UMHL-quantified MHBs as classifiers for MVIand MVI+ samples. Combining the MHBs' methylation scores as classifiers and applying RF method, we trained and cross-validated MVI- and MVI+ classification models. We found the AUC of these models is $85.9 \%$ (CI: $83.5 \%$ $88.3 \%)$.

Conclusions These data showed that DNA methylation signatures can provide outstanding diagnostic accuracy for HCC and MVI.

\section{IDDF2019-ABS-0320 TERMINALIA ARJUNA BARK EXTRACT PROTECTS AGAINST EARLY ALCOHOL- INDUCED LIVER INJURY IN THE RAT}

Ramesh Kumar Meena*, Tejpal Singh. Department of Biological Sciences, University of Rajasthan, Jaipur, India

\subsection{6/gutjnl-2019-IDDFabstracts.109}

Background Terminalia arjuna, an indigenous plant used in ayurvedic medicine in India, primarily as a cardiotonic is also used in treating diabetes, anemia, tumors and hypertension. Oxidants have been shown to be involved in the alcoholinduced liver injury. Arjunolic acid (AA) is an oleanane triterpenoid found mainly in the heartwood of Terminalia arjuna. This study was designed to determine whether Terminalia arjuna bark (TAB) extract, composed mostly of ellagitannins oligomers, protects against early alcohol-induced liver injury in rats.

Methods Total fifty-eight male Wistar rats were fed high-fat liquid diets with or without ethanol (10-14 g/kg per day) and $\mathrm{TAB}$ extract $(300 \mathrm{mg} / \mathrm{kg}$ per day) continuously for $\underline{4}$ weeks using an enteral feeding protocol.

Results Mean body weight gains (approximately $4 \mathrm{~g} /$ day) were not significantly different between treatment groups. TAB extract did not affect average daily urine ethanol concentrations (approximately $200 \mathrm{mg} / \mathrm{dL}$ ). After $\underline{4}$ weeks, serum alanine amino transferase levels of the ethanol group were increased nearly fourfold $(110 \pm 16 \mathrm{IU} / \mathrm{L})$ compared to control values $(35 \pm 3 \mathrm{IU} / \mathrm{L})$; this effect of ethanol was blocked by $\mathrm{TAB}$ extract $(60 \pm 6 \mathrm{IU} / \mathrm{L})$. Additionally, enteral ethanol caused severe fat accumulation, mild inflammation, and necrosis in the liver; TAB extract significantly blunted these changes. Increases in liver TNFalpha protein levels caused by ethanol were completely blocked by TAB extract. Further, ethanol significantly increased the accumulation of protein adducts of 4hydroxynonenal, a product of lipid peroxidation serving as an index of oxidative stress; again this was counteracted by the addition of TAB extract.

Conclusions The result indicates that the TAB extracts exhibit the antioxidant activity through correction of oxidative stress and validates the traditional use of Terminalia arjuna to prevents early alcohol-induced liver injury.

\section{IDDF2019-ABS-0325 SUPERIOR EFFICACY AND LONG-TERM SURVIVAL BENEFIT OF HDAC8 AND PD-L1 CO-BLOCKADE IN LIVER CANCER IMMUNOTHERAPY}

${ }^{1}$ Weiqin Yang*, Yu Feng, ${ }^{1}$ Jingying Zhou, ${ }^{1}$ Otto KW Cheung, ${ }^{2}$ Hanyong Sun, ${ }^{3}$ Stephen L Chan, ${ }^{4}$ Anthony WH Chan, ${ }^{5}$ Zhiwei Chen, ${ }^{6}$ Kevin YL Yip, ${ }^{4}$ Ka-Fai To, ${ }^{2}$ Joseph JY Sung, ${ }^{1}$ Alfred SL Cheng. 'School of Biomedical Sciences, The Chinese University of Hong Kong, Hong Kong; ${ }^{2}$ Department of Medicine and Therapeutics, The Chinese University of Hong Kong, Hong Kong; ${ }^{3}$ Department of Clinical Oncology, The Chinese University of Hong Kong, Hong Kong: ${ }^{4}$ Department of Anatomical and Cellular Pathology, The Chinese University of Hong Kong, Hong Kong; ${ }^{5}$ AIDS Institute, The University of Hong Kong, Hong Kong; ${ }^{6}$ Department of Computer Science Engineering, The Chinese University of Hong Kong, Hong Kong

\subsection{6/gutjnl-2019-IDDFabstracts.110}

Background The heterogeneous responses to immune-checkpoint blockade (ICB) therapy e.g. anti-programmed deathligand 1 (PD-L1) antibody are attributable to the complex interplay between a range of cancer-cell-autonomous cues and immunosuppressive tumor microenvironment. Two recent phase I/II trials of $\mathrm{PD}-1$ checkpoint inhibitors in patients with advanced hepatocellular carcinoma (HCC) have produced promising results, yet the objective response rates were relatively low $(<20 \%)$. Accumulating evidence underscore the fundamental importance of epigenetic regulation in tumor immune evasion. We have previously elucidated a critical role of histone deacetylase 8 (HDAC8) in hepatic carcinogenesis (Cancer Research 2015;75:4803-16). Here, we aim to investigate the therapeutic potential of a HDAC8-specific inhibitor PCI-34051 in preclinical HCC models.

Methods The effect of PCI-34051 on tumorigenicity was investigated in orthotopic HCC mouse models. Immune profiling in tumor microenvironment was determined by multi-color flow cytometry. The underlying mechanism was investigated by integrative epigenomics analysis. The anti-tumor efficacy of combined therapy with PCI-34051 and anti-PD-L1 antibody was further determined.

Results PCI-34051 significantly suppressed tumorigenicity in immunocompetent but not immunodeficient mice, which was accompanied by increased effector $\mathrm{T}$ cell tumor infiltration. Mechanistically, tumor-intrinsic HDAC8 epigenetically silenced chemokine CCL4 expression via H3K27 deacetylation at the enhancer to inhibit $\mathrm{T}$ cell infiltration to tumor. Furthermore, PCI-34051 dramatically improved the therapeutic efficacy of 
anti-PD-L1 checkpoint blockade, leading to eradication of large tumor. More importantly, HDAC8 and PD-L1 co-blockade resulted in long-term survival (more than 1 year) with the induction of $\mathrm{T}$ cell memory responses.

Conclusions Our finding delineates that selective chromatin modifications by HDAC8 can augment the therapeutic efficacy of PD-L1 blockade therapy to fully unleash $\mathrm{T}$ cell responses, leading to long-term remission of HCC. This study highlights a new epigenetic target for immune potentiation in HCC, providing a rational combinatorial epigenetic immunotherapy.

\section{IDDF2019-ABS-0326 EVALUATION OF THE EFFECTS OF THE HYDROALCOHOLIC EXTRACT OF LIV.52 DS ON PARACETAMOL INDUCED LIVER TOXICITY AND OXIDATIVE STRESS IN RATS}

Arun Gandhi*, Priya Kakkar. Faculty of Life Sciences, Maharshi Dayanand University, Rohtak, Haryana, India

\subsection{6/gutjnl-2019-IDDFabstracts.111}

Background Oxidative stress induced by toxicants is known to cause various complications in the liver. Herbal drug such as Liv.52 is found to have a hepatoprotective effect. However, the biochemical mechanism involved in the Liv.52 DS mediated protection against toxicity is not well elucidated using suitable in vivo models. Paracetamol causes oxidative stress and dysfunction of the liver.

This study was undertaken to evaluate the effects of the hydroalcoholic extract of Liv.52 DS on some biochemical and histopathological parameters of liver tissue in against paracetamol-induced hepatic damage in rats.

Methods Wistar rats were orally administered with $2 \mathrm{~g} / \mathrm{kg}$ body weight Paracetamol. Vehicle (distilled water) and silymarin $(50 \mathrm{mg} / \mathrm{kg}$ body weight) was used as the negative and positive control groups, respectively. Paracetamol-administered groups were treated with Liv 52 DS extract (100, 200, and $400 \mathrm{mg} / \mathrm{kg}$ ). After 15 days of treatment, the blood specimens and liver samples were examined. Alteration in the levels of biochemical markers of hepatic damage like AST, ALT, ALP and lipid peroxides were tested, and phytochemical tests were also performed.

Results In Paracetamol-treated group, the levels of serum urea, high-density lipoprotein (HDL), and liver superoxide dismutase (SOD), catalase (CAT), and vitamin C significantly decreased $(\mathrm{p}<0.05)$ compared to control. Also, in this group, serum triglyceride (TG), total cholesterol (TC), very low-density lipoprotein cholesterol (VLDL), protein carbonyl (PC), malondialdehyde, tumor necrosis factor- $\alpha$ (TNF- $\alpha$ ), and TNF$\alpha$ gene expression significantly increased $(\mathrm{p}<0.05)$ as compared to the control (vehicle-treated rats). Treatment with Liv. 52 DS extract in a significant increase $(\mathrm{p}<0.05)$ in CAT, SOD, vitamin $C$, HDL and a significant decrease $(p<0.05)$ in the level of urea, MDA, PC, TG, TC, VLDL, TNF- $\alpha$ protein, and the gene expression of TNF- $\alpha$ compared with the test without treatment group. Histopathological evidence demonstrated that treatment with Liv.52 DS extract could decrease liver lymphocyte infiltration.

Conclusions The present study suggests that Liv. 52 DS extract possesses hepatoprotective activity. It could be an effective and promising preventive agent against Paracetamol-induced hepatotoxicity.

\section{IDDF2019-ABS-0334}

CLINICAL ESCHERICHIA COLI NF73-1

ISOLATED FROM A PATIENT WITH NONALCOHOLIC STEATOHEPATITIS INDUCES LIVER INJURY THROUGH IMPAIRING INTESTINAL BARRIER FUNCTION AND INDUCING INFLAMMATORY RESPONSES OF HEPATOCYTES

Yang Song*, Zhe Wu, Jun Xu, Yujing Chi, Yifan Zhang, Yulan Liu. Peking University People's Hospital, China

\subsection{6/gutjnl-2019-IDDFabstracts. 112}

Background Gut microbiota plays critical roles in nonalcoholic fatty liver disease (NAFLD). We have previously isolated and identified one clinical Escherichia coli (E. coli) strain from the intestinal mucosa of a nonalcoholic steatohepatitis (NASH) patient, and named it as E. coli NF73-1. Our aim is to investigate the role of E. coli NF73-1 in the development of NAFLD in a high-fat diet (HFD) mice.

Methods Conventional (CV) mice, plus mice treated with antibiotics $(\mathrm{AB})$ to deplete gut microbiota, were fed with HFD for 12 weeks. At the $10^{\text {th }}$ week, mice were treated daily with oral gavage of LB, live-NF73-1, or pasteurized-NF73-1 (pas-NF73-1) for 2 weeks. $\mathrm{AB}$ mice were treated in drinking water containing $1 \mathrm{~g} / \mathrm{L}$ ampicillin, $500 \mathrm{mg} / \mathrm{L}$ vancomycin, $1 \mathrm{~g} / \mathrm{L}$ neomycin, and 1 $\mathrm{g} / \mathrm{L}$ metronidazole for 4 weeks, starting at $6^{\text {th }}$ week. In vitro bacterial translocation and transepithelial permeability assay was performed. Primary hepatocytes from NAFLD mice were cocultured with NF73-1 to evaluate inflammatory responses.

Results Live-NF73-1 group developed severer liver pathology than $\mathrm{LB}$ and pas-NF73-1 groups in both $\mathrm{CV}$ and $\mathrm{AB}$ mice, verified by increased NAFLD activity (NAS) score. Besides, intestinal permeability was higher in live-NF73-1 group than that in LB and pas-NF73-1 groups of both $\mathrm{CV}$ and $\mathrm{AB}$ mice, supported by decreased expression of ZO-1 and Occludin in the colon. In vitro bacterial translocation and transepithelial permeability assay indicated that HT-29 cells treated with live-NF73-1 developed a higher concentration of translocated bacteria and FITC fluorescein than LB and pas-NF73-1 groups. Interestingly, live-NF73-1 decreased mRNA levels of ZO-1, Occludin and Claudin2 in Caco 2 cells, and downregulated mRNA expression of Claudin2 and E-cadherin in HT-29 cells compared with LB and pasNF73-1 groups. NF73-1 also induced inflammatory responses of primary hepatocytes, supported by increased IL-6 expression.

Conclusions Clinical E. coli NF73-1 aggravates liver injury in NAFLD mice, through impaired intestinal integrity and inflammatory responses in hepatocytes. These findings provide new insights on management using specific bacterial strain.

\section{Clinical Gastroenterology}

\section{IDDF2019-ABS-0013 CELIAC CRISIS IN AN ADULT TYPE 1 DIABETES MELLITUS PATIENT PRESENTING WITH DIARRHEA, WEIGHT LOSS AND HYPOGLYCEMIC ATTACKS- A RARE ENTITY}

Shivam Shivam*, Pratibha Nadig, Vijaya Sarathi, Manohar KN. Vydehi Institute of Medical Sciences and Research Centre, India

10.1136/gutjnl-2019-IDDFabstracts. 113

Background Type 1 diabetes mellitus (T1DM) is an autoimmune disease, characterized by loss of insulin-producing beta 